\title{
Characteristics of Ying Shan formation Carbonate Sequence Stratigraphy in middle of the Tarim Basin
}

\author{
Jianfa Han1, Hao Li², Xiaoyong Hu1, Qinglong Wang2, Yubin Song1 \\ 1Research Institute of Exploration and Development,PetroChinaTarim Oilfield Company,Kuerle, \\ China
}

2School of OceanSciences and Resources, China University of Geosciences, Beijing, China

\begin{abstract}
Keywords: Tarim Basin, Ying Shan formation, Sequence, systems tract style
\end{abstract}
\begin{abstract}
The Ying Shan formation carbonate systems is identified 11 kinds of sequence boundary characteristics ,generalized3 genetic types of sequence boundary and divided into 5 third-order sequence(Sq1-Sq5) to establish sequence stratigraphic framework in Ying Shan formation , Tarim Basin by the integrated analysis of many seismic lines, drilling cores and well logging data.Yi Jianfang formation is included Sq5 which was widely corroded with the top of Ying Shan formation in Ta Zhong area.3 kinds of sedimentary systems tract style, including "HST+TSTorHST" and "LST+TST+HST" from different tectonopalaeo geographic backgrounds are inducted. The former is more common than the latter in which LST is composed by graded bedding grain limestone with debris flow.
\end{abstract}

\section{INTRODUCTION}

The establishment of carbonate sequence stratigraphy plays an important role on the prediction of favorable reservoir distribution. Recent research results show that different levels of sequence boundary play important roles in controlling the scale of the development of Karst[Yu Bingsong,2005]. So the study on the sequence boundary characteristics, genetic types and boundary style is important for understanding the evolution of tectonic-sedimentary in Tazhong area Yingshan formation and the further exploration and development.

Previous research has made the corresponding results in sequence boundary characteristics and characteristics of system tract, structure style, law of sea level change and palaeontological characteristic in Ordovician Carbonates in TarimBasin[Lin Changsong,2011,2012][Wang Hongyu,2008], but there are few detailed studies on specific Yingshan formation, and sequence division scheme is different and confusing, and there is no common conclusions and understanding. In this paper, we studied in the sequence boundary characteristics, genetic type and the sequence style of the Yingshan formation based on a detailed analysis of the outcrops, log data (especially the imaging logging), and seismic data, and then, made the division scheme of Yingshan formation carbonate sequence.

\section{TECTONIC SETTING}

The Tarim Basin, located in western China, is a large superimposed basin that underwent multiple phases of tectonic deformation from the Sinian to Cenozoic. The basin basement is differentiated, and tectonic paleogeography changed several times, and plate tectonic setting is complex. The basin architecture is complicated, and is characterized by the development of a series of large-scale tectonic unconformities and paleo-uplifts, such as Tabei, Tadong,Tazhong and Bachu uplift. 


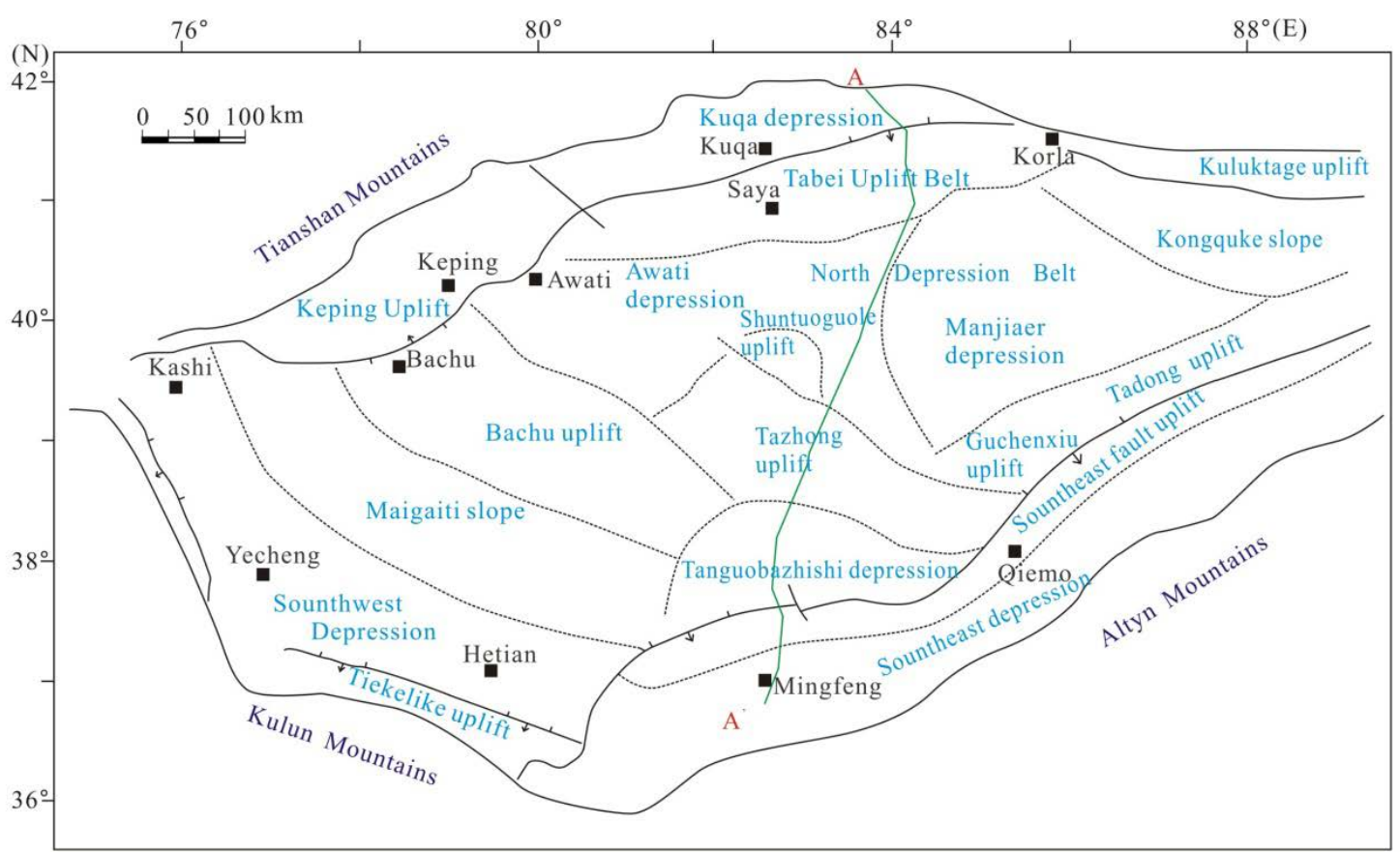

Figure 1. Schematic tectonic map of the Tarim Basin, showing the distribution of tectonic units within the basin

The study area is mainly located in the Tarim Basin Tazhong paleo-uplift, affected in the Caledonian movement. The paleouplift occurred in the Middle Ordovician.Tarim Basin changed from the extension to the extrusion environment, and North-South differentiation turned to East-West differentiation. The thrust movement of Tazhong I fault was NE oriented. The NW trending structures of Tazhong uplift began to form[10]. Strong uplift and erosion in the area produced a wide range of sedimentary discontinuity, lacking the Yijianfang formation of Middle Ordovician and theTumuxiuke formation of the lower part of Upper Ordovician,and forming a large area of crust of weathering karst reservoir. At the end of Late Ordovician the fold andpaleoupliftwas forming, and the basic pattern of the Tazhong complex anticline formed.

\section{THE COMPOSITION CHARACTERISTICS OF STRAGIGRAPHY AND FZCIES}

The thickness of Yingshan formation in the middle-lower Ordovician in Tazhong is about 300-700 meters. Yingshan formation is divided into four members. The thickness of Ying4 member is about $90 \mathrm{~m}$, mainly composed of aplite dolomite and dolarenite. The Ying 3 member is about 140 meters thick, and the content of dolomite decreased compared with Ying4 member, mainly composed of sparrycal care nite grain stone and micrite interbedded medium-thin aplite dolomite. The Ying3 member is about 160 meters thick, which isintraclast beach sediment, mainly composed of thick intraclasticgrainstone and argillaceous limestone. The Ying1 member is about 80 meters thick, on which is a crust of weathering unconformity with Yijianfang formation, mainly composed of light gray medium-thick argillaceous limestone, algal limestone interbedded medium-thin intraclasticgrainstone and wackestone as well as bioclastic shoal deposition on the top.

\section{THE CHARACTERISTICS OF SEQUENCE STRATIGRAPHIC STRUCTURE AND SEDIMENTARY SYSTEM OF YINGSHAN FORMATION IN TAZHONG}

On the basis of 3D seismic sections across the Tazhong area, and the comprehensive analysis of drilling and core, we identified5 tertary sequence boundaryin the Yingshan formation , which is bottom boundary of Yingshan formation ( SB1), bottom boundary of Ying3 member (SB2), bottom boundary of Ying2 member (SB3), upper of Ying2 member (SB4), middle-lower of Ying1 member

(SB5). In outcrops in Bachu and Keping, sequence boundaries are basically consistent with each member ones. According to the characteristics of evolution of the basin, on the basis of the genetic 
types and forms of Yingshan formation, the sequence boundary was divided into 4 types.

On the basis of the divided type of sequence interface, the internal architecture of the Yingshan and Yijianfang Formation in Tazhong area consists of five third-order depositional sequences from the base upward: Sq1, Sq2, Sq3, Sq4, Sq5. Sq5 includes overlying Yijianfang Formation.

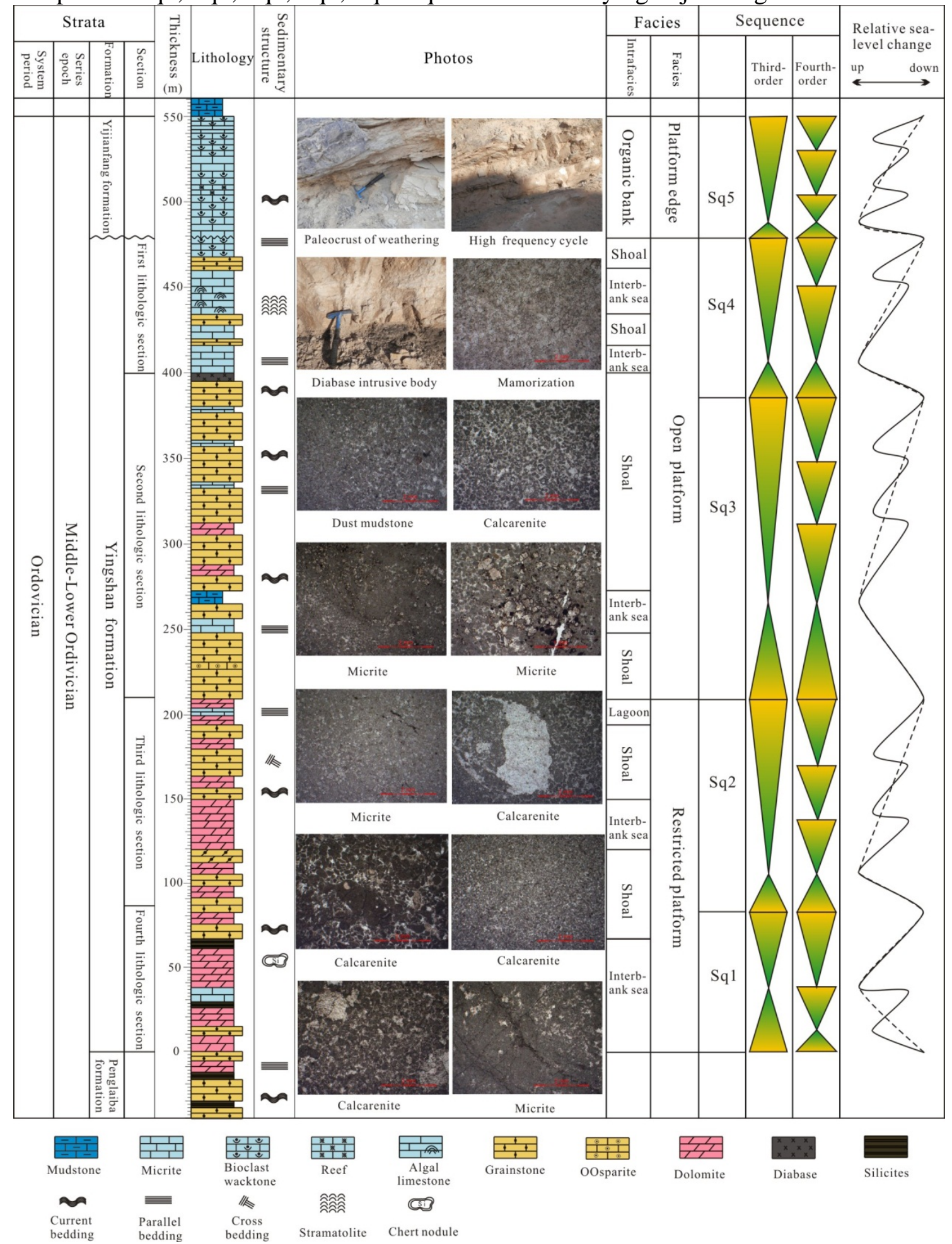

Figure 2. Integrated outcrop figure of Ba Chu from Yingshan formation tu Yi Jianfang formation

The bottom interface of the Sq1 is the boundary of the Yingshan Formation and the underlying Penglaiba Formation. Itis a type of localexposure interface, and it can be tracked in the whole area. The seismic cross section shows that the seismic reflection features of the interface are intermittent and different strength of amplitude. Those features reflectthepresence ofkarst phenomenon. Seismic section of westTazhong area reveals onlap contact relationship. Tazhong 162 well has LSTslumpbreccia,calcrudite which has lenticular random structure, and bottom's scoured surface 
(figure 3-f). In the Keping outcrops which is located in the western margin of the basin, we can recognize significantexposurekarst and the ancientsoilfeatures ontheinterface (figure 3-a). Besides, rockcolorschange significantly in the vicinity of the interface. The colors change from light grey to browngrey from bottom to up (figure 3-b). Generally, Sq1isasedimentary cycle from localtransgressiveonlap to progradingregression.

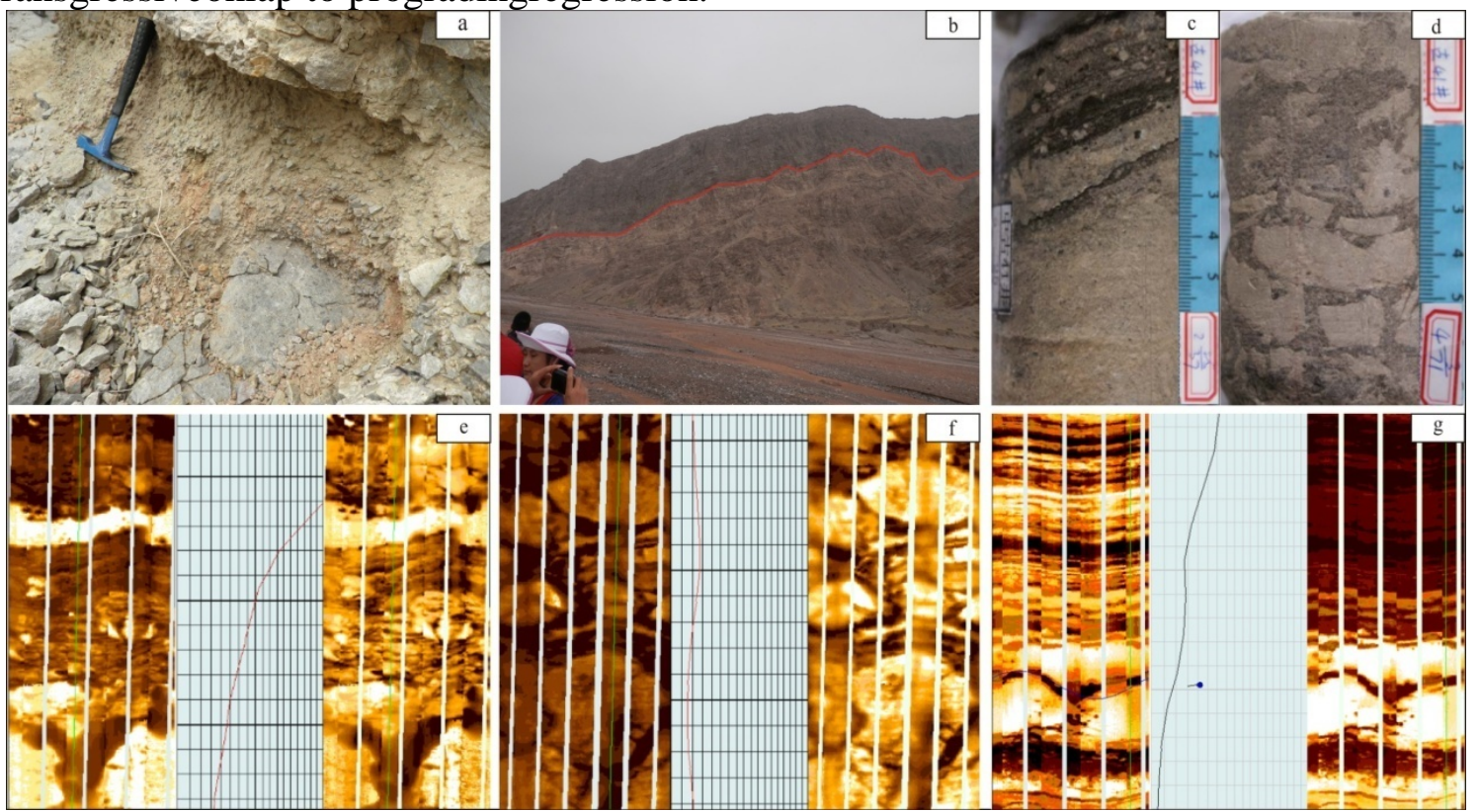

Figure 3.The Sequence boundary representation of Yingshan formation in Tarim Basin

The bottom interface of the Sq2 is located in the vicinity of the boundary of the third and the fourth lithologic section of the Yingshan Formation. It is a type of local exposure interface, and roughly made up of Yingsan Formation. In Tazhong area, the main contact relationship is conformable contact. There is uncomformable contact only in the midwest and eastern swelling areas. Tabeipalaeohigh ramp region consists of there to four lithosomic bodies which have onlap structures, and shows retrograding structure overall which represents the slow rise of sea level.

The bottom boundary of the Sq3 is territorial transgressive onlap unconformity interface. It shows significant onlapuncomformable contact relationship in the entire Tazhong overlay area's seismic sections, and truncation unconformity in local areas. The top boundary of the Sq3 is significant exposure level. Compared with Sq1 and Sq2, the lithologies of Sq3 change from dolomitic limestone to limestone.

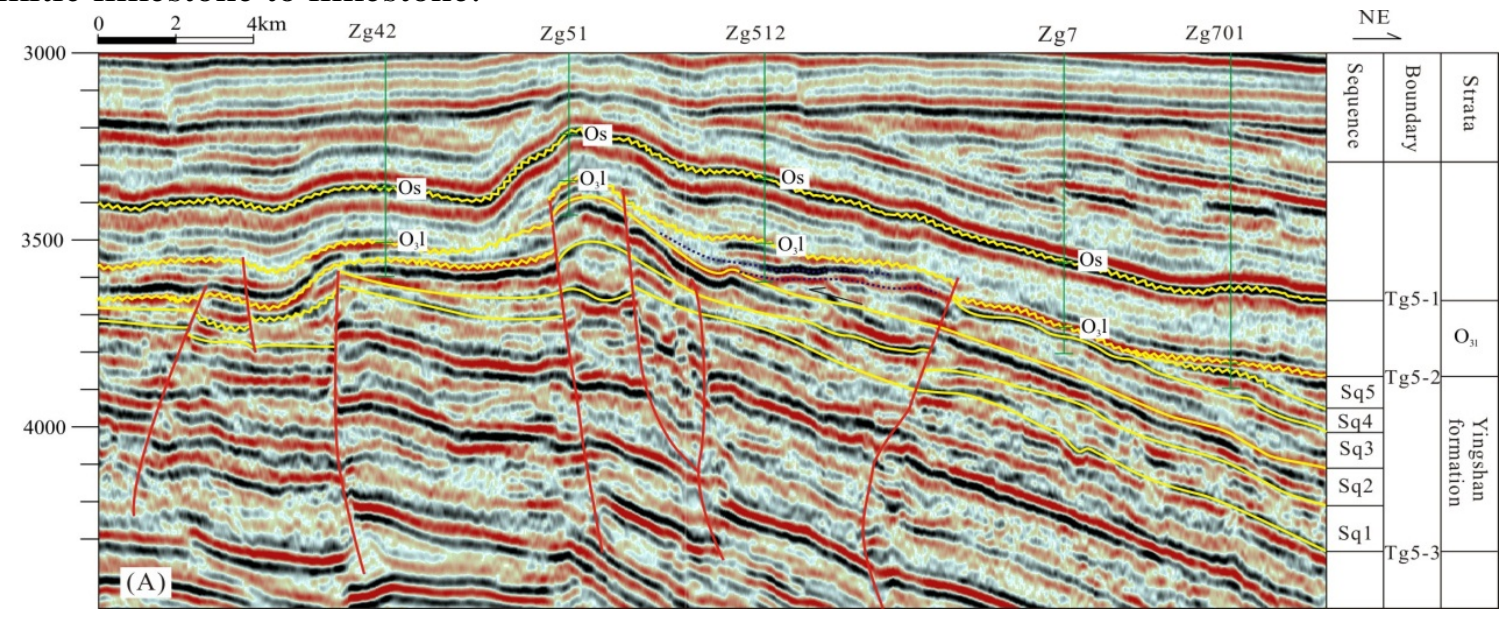

Figure 4. The stratigraphic framework explaination section of Yingshan formation in easternTazhong uplift

The bottom boundary of SQ4 belongs to local exposure-type sequence boundary,the erosion of eastern and western Tazhong area to underlying strata is weak, middle is strong,the low slope along the platform or ramp margin is wedge-shaped sedimentary bodies,internal display onlapstrcture,the part of western Tazhong area exist the phenomenon of onlap.

The sequence units of SQ5 in western basin mostly develop YijianfangFormation,the top of this 
sequence in Tazhong area is the unconformity interface called Tg52 which presents obvious angular unconformity with the underlying strata,it is a typical erosion surface of unconformity by tectonic uplift,it develops 1 or 2 sets of independent karst system from 1 to 20 meters under this sequence boundary,which the GR value is definitely too high(Fig.3-e).

According to the fine analysis of outcrop,well logging curve and imaging logging,it indicates that the composition of systems tracts of carbonate sedimentary sequence formed in different tectonic paleogeographic background exists obvious differences.We conclude three types of sequence depositional architecture in YingshanFormation,the development and distribution is mainly controlled by tectonic paleogeographic background.

The type of sequence architecture developed in restricted platform: sequence structures feature the asymmetric development pattern of "HST+TST" or "HST+HST",this configuration which mainly controlled by the production rate of carbonate and the sea-level change is mainly developed in the bottom boundary of SQ1,SQ2 and SQ3.

The type of sequence architecture developed in open platform: sequence structures feature "HST+TST" which mainly controlled by the production rate of carbonate and the sea-level change is mainly developed in the bottom boundary of SQ4 and SQ5.

The type of sequence architecture developed in wedge shelf of low water levels: sequence structures obviously feature "LST-TST-HST", LST composed by debris flow grainstone of graded bedding,mainly controlled by the sea-level change and the deposit velocity,it is mainly developed in the bottom boundary of SQ1,SQ3 in part regions and the top or bottom boundary of SQ5.

\section{CONCLUSION}

Yingshan and Yijianfang formation can be divided into 5 third-order sequence (Sq1-Sq5), in which Sq1-Sq4 can equal to the Yingshan formation lithologic section boundary, Sq5 include Yijianfang formation in western Tarim basin. Sequence boundary of Tazhong uplift is different from the western Tarim basin.Sq1, Sq2 can equal to the third and fourth lithologic section, Sq3 include the up and lower of second lithologic, Sq4 is the top of second lithologic section and bottom of the first lithologic, Sq5 contains top of the second lithologic section and Yijianfang formation which was widely eroded in Tazhong area. Every sequence stratigraphical unite shows the different sedimentary structure from retrogradation to progradation or aggradation. Thick packstone, intraclasticgrainstone are common in the early transgression or late highstand systems tract, while micrite, bioclastwackstone, algal limestone mainly appear in the late transgression or early highstand systems.

4 kinds of sequence boundary has been concluded by the evolution characteristic of Tarim basin which includes tectonic uplift erosion unconformity surface, transgressive onlap sequence boundary, local exposure sequence boundary and drowned unconformity(Sq1, Sq2, Sq4, Sq5). The bottom of Sq3 is a typical transgressive onlap sequence, the top of the Sq5 is a drowned unconformity in western basin, while, it shows tectonic uplift erosion unconformity surface in tazhong area.

The evolution of Yingshan formation mainly changes from restricted platform to open platform and platform margin. Different kinds of sedimentary composition type develop in different sedimentary environment. 3 kinds of composition type ("HST+TST", "HST+TST" or "LST-TST-HST") can be identified, TST in the restricted platform mainly develops micrite, and HST mainly includes dolomite, limestone with variable contents of dolomite and grainstone, While, thick grainstone, bioclastgrainstone, packstone always appears in the open platform highstand systems tract. LST mainly composed by grainstone debris with graded bedding which is the result of event depositional process. Tectonic paleogeographic background mainly controls their distribution.

\section{ACKNOWLEDGMENTS}

This work is the result of many years of research on the Tarim Basin and was supported by National Science and Technology Major Project (2011ZX05001-002-003). 


\section{REFERENCES}

1. YU Bingsong,et al. 2005. Sequence Stratigraphy Framework and Its Control on development of Ordovician Carbonate reseivoir in Tarimbasin[J],Oil \&Gas Geoleogy(26): 305-316

2. Changsong Lin, etal. 2011. Squence architecture and depositional evolution of the Ordovician carbonate platform margins in theTarim Basin and its response to tectonism and sea-level change[J]. Basin Research24:1-24

3. Changsong Lin, et al. 2012. Distribution and erosion ofthe Paleozoic tectonic unconformities in the Tarim Basin, Northwest China: Significance for the evolution of paleo-uplifts andtectonic geography during deformation.Journal of Asian Earth Sciences46:1-19

4. LIN ChangSong, et al. Tectonic framework and paleogeographic evolution of the Tarim basin during the Paleozoic major evolutionary stages[J].ActaPetrologicaSinica27(1) :210-218

5. Changsong Lin, et al. 2012. Major Unconformities, Tectonostratigraphic Framework, and Evolution of the Superimposed Tarim Basin, Northwest China[J].Journal of Earth Science23(4):395-407

6. WANGHongyu, et al .2008. Study of the method for identification of carbonatelogging sequence stratigraphy-a case study of the Ordovician in the Ka1area of Tazhong uplift, Tarim basin. Earth Science Frontiers 15( 2) : 52-58 